\title{
QUALITY MANAGEMENT OF INPATIENT MEDICATION ADMINISTRATION IN HONG KONG PUBLIC HOSPITALS
}

\author{
Catherine K. Y. Kwong', Ben Y. F. Fong' \\ 1. School of Professional Education and Executive Development, The Hong Kong Polytechnic University
}

Correspondence: kwongkiuyan@gmail.com

\section{ABSTRACT}

Medicine maladministration can result in various side effects to patients, including serious complications, extended medical care, incapacity and death. In Hong Kong public hospitals, the pattern of medication error consists of prescribing error usually made by physicians, dispensing error resulting from pharmacists and dispensers, drug administration error caused by nurses and patientcare workers and technology-related error associated with technology used in the drug administration process. Medication Administration Record (MAR) and ward stock are the usual inpatient medication system within the Hospital Authority public hospitals before the development of the electronic system, while the Inpatient Medication Order Entry (IPMOE) functions to provide real-time accessibility in patients' medication profiles by different professions and health units. However, several factors are related with medication errors. For instance, the ambiguous handwriting orders in the MAR prescribed by physicians affect the transcription by pharmacists and the administration procedures in ward by nurses. Administering medicines in ward stock before pharmacists vetting increase the chance of making errors. Poor interface issues between users and system, and the computer overreliance also contributed to technology-related errors. In order to reduce the occurrence of medication incidents, implementation of Automated Pharmacy Distribution Systems helps moving towards a closed loop medication management system. Modifying technologies in barcode assisted medication administration and enhancing the IPMOE with a pop-up message could help intercepting drug administration errors to patients. The elimination of the potential risks arising from the prescribing, dispensing and drug administration processes brings the achievement of medication safety in Hong Kong public hospitals.

\section{KEYWORDS}

quality management, medication error, inpatient drug administration, public hospitals

\section{INTRODUCTION}

Medication maladministration to patients can lead to serious adverse drug events, prolonged hospitalisation, extra medical treatment, morbidity and death as well.[1] In public hospitals, the medication system should be efficient and effective to ensure the accuracy of prescribing and dispensing medicines to the right patients. Unsafe dispensing practices and medication errors are undesirable clinical practice. There are potentially preventable factors to achieve the medication safety goal.[2] In order to reduce the occurrence of medication errors inside the hospitals, it is essential to find out the frequently undetected errors and the associated factors causing the problems in the whole medication management process. The elimination of the potential risks arising from the prescribing, dispensing and administration procedures of drugs is the concrete action to minimize the hazards to the patients. This paper aims to review the current practice of inpatient medication administration in Hong Kong public hospitals, to examine factors leading to medication incidents and to 
propose operational strategies to improve inpatient medication administration.

\section{STATISTICS IN HONG KONG}

The Serious Untoward Event (SUE) Policy has been implemented in Hong Kong public hospitals since 2010 in co-operation with the Sentinel Event (SE) Policy starting in 2007.[3] SUE includes untoward medication errors and patient misidentification that can lead to death or permanent harm. According to the annual report of the Hospital Authority (HA), 72 SUE were reported from the fourth quarter in 2018 to the second quarter in 2019 (Fig. 1).[3] The proportion of medication error was much greater than patient misidentification. The three most common issues in untoward medication errors came from prescriptions of known drug allergies, dangerous drugs and anticoagulants.[3] It showed that the errors in prescribing procedures should be mostly focused and solved with some effective interventions.

FIGURE 1: YEARLY DISTRIBUTION OF SUE BY CATEGORY [3]

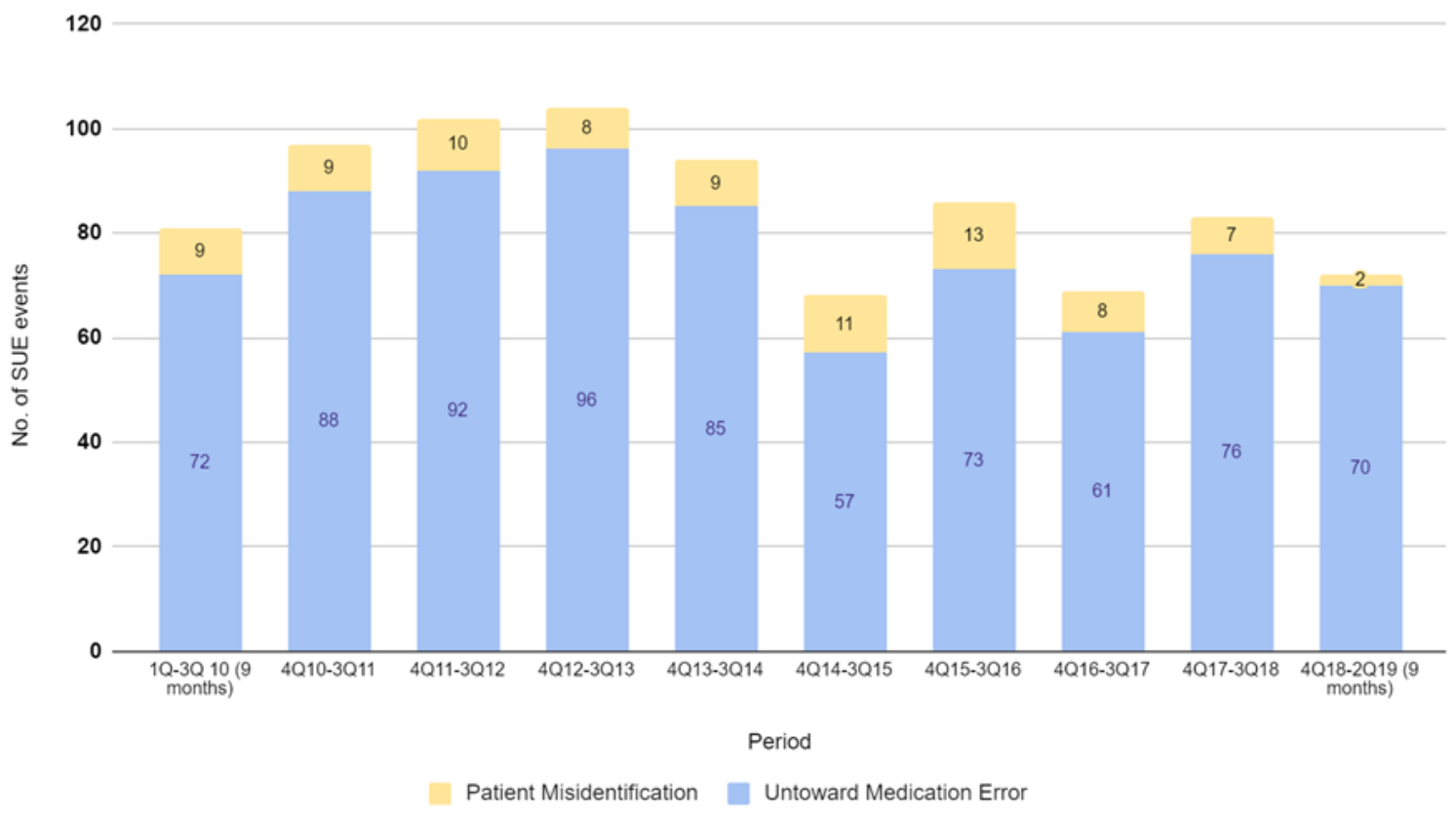

\section{CLASSIFICATION OF ERRORS IN MEDICATION} INCIDENTS

Medication errors may arise from both human or system failures.[4] The pattern of medication errors in Hong Kong public hospitals consists of $53.4 \%$ prescribing error, $29.0 \%$ drug administration error, and $17.6 \%$ dispensing error.[1] The 'Swiss Cheese Model' is used to explain the interception of human errors.[5] In the healthcare system, doctors, pharmacists and nurses are considered as defensive layers in the medication use process corresponding at the prescribing, drug dispensing and drug administration stages respectively. The study indicates that the prescribing errors and dispensing errors could be partially intercepted by hospital pharmacists and nurses, while majority of patient-reached administration errors are unnoticed or not intercepted.[1] There are also technology-related errors in the system usage.

\section{PRESCRIBING ERRORS}

These errors are made by doctors.[6] The errors may occur as a result in a prescribing writing process or a medication ordering decision. They include incorrect choice of drugs, incorrect dosage, wrong route and frequency of administration, incorrect administration instructions of a drug product, drug allergies, undesirable drug-drug interactions. However, in the inpatient system of the HA public hospitals, some prescribing errors are likely to reach the patient undetected, including wrong instructions, drug omission, double entry, wrong duration, known drug allergy and wrong patients. [1] 


\section{DRUG ADMINISTRATION ERRORS}

The most frequently non-intercepted errors are drug administration errors made by nurses and patient-care workers. Usually such errors happen when there is deviation from the physician's orders. [6] The safety of the medication administration process is affected due to dose omission, extra dose, wrong drug administration, wrong patient, wrong strength of drug, wrong time, wrong flow rate of intravenous fluid and drug allergy. [1]

\section{DISPENSING ERRORS}

The process of dispensing from dispensers is integrated with patient counselling from pharmacists. It is important to maintain good quality in the sequence of steps to produce best practice and outcomes for the patients. Dispensing errors are made by pharmacists and dispensers when distributing medications to outpatients or inpatients. [6] The most frequent errors include wrong dilution volume in drug preparation, incorrect labelling and directions, and drug dispensed to the wrong patient.[1]

\section{TECHNOLOGY-RELATED ERRORS}

These errors are associated with a technology used in the drug administration process. [7] They are divided into the two categories of socio-technical errors and device errors. A socio-technical error is an unintended and unexpected result due to a human and technology interaction. The unintended errors are produced by health care providers, like selecting a wrong drug name from the electronically prescribing system. A technical failure of the device is not related to human action, for example, an unanticipated device error occurs when a defected infusion pump is used resulting in a wrong dose of parenteral infusion being given.

\section{DRUG DISTRIBUTION SYSTEMS IN PUBLIC HOSPITALS}

\section{INPATIENT DRUG PRESCRIBING SYSTEM - MANUAL SYSTEM}

Before the full development of the electronic system, the general process of handling inpatient medication is the Medication Administration Record (MAR).[8] Inpatient prescribing by doctors is hand-written on the MAR form. Pharmacists need to transcribe the handwriting on MAR to the electronic system in pharmacy. Medications for inpatients are then prepared and distributed in a central pharmacy. For patient-specific unit doses, those drugs would be sent to the wards daily. The medications are transported from the pharmacy to the wards for administration by nurses as described in (Fig. 2).

FIGURE 2: FLOWCHART OF INPATIENT MEDICATION ADMINISTRATION PROCESS (MAR)
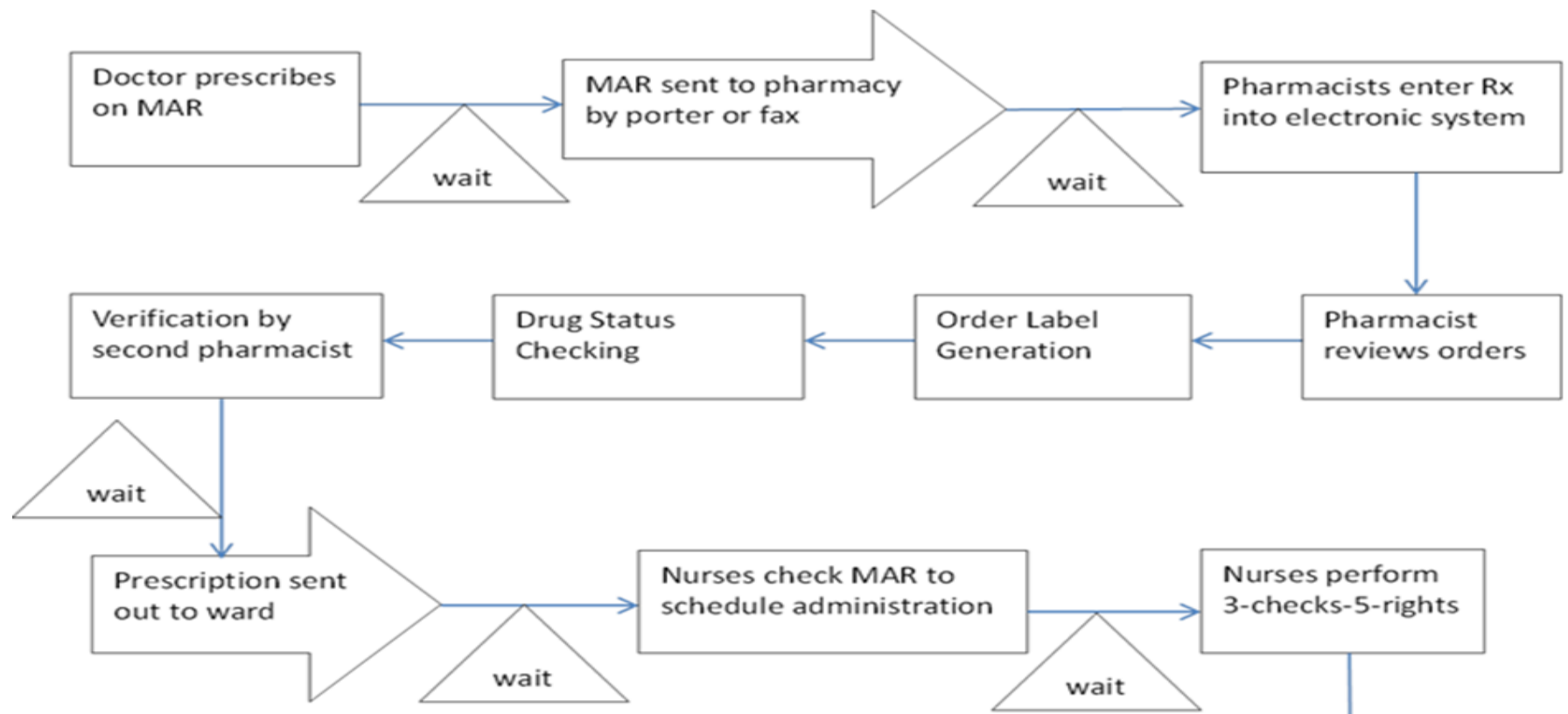

Nurses perform

3-checks-5-rights

Patient administer

medication

Nurses sign on

MAR

Source: [8] Chiang SC. In-Patient Medication Order Entry System - contribution of pharmacy informatics [Internet]. 2015 [cited 2019 Nov 5]. Available from http://www3.ha.org.hk/haconvention/hac2015/proceedings/downloads/s 12.1.pdf 


\section{INPATIENT DRUG ADMINISTRATION SYSTEM - WARD STOCK}

Apart from manual MAR, nurses need to manage the drugs stored in the wards. An advanced technical system, Barcoded Ward Stock Topping Up System, is used to extend the pharmacy functions in the wards at Ruttonjee Hospital, a district general hospital.[9] All drugs stored in cabinets and ward fridges are barcoded and kept in the ward according to the pre-agreed ward stock list and quantities. With the help of barcode device, pharmacy staff can capture and replenish items before the minimum level is reached without nurse requisition. It can better control the ward stock and storage conditions, compared with traditional ward stock procedures.

\section{INPATIENT DRUG ADMINISTRATION SYSTEM - INDIVIDUAL PATIENT DISPENSING}

Individual Patient Dispensing system in wards is used concurrently when a course of therapy is dispensed according to the manual prescription for each patient. A specific medication profile could be maintained after reviewing the appropriateness of the therapy by pharmacist. An individual drug supply for several days of therapy is sent to wards and it helps to limit the time intervals for dispensing. Computerized Automatic Refill System is an electronic system to save the previous dispensed drug in record and repeat the therapy when necessary. Nurses are required to inform pharmacy if there is a change in patients' complete drug profile. This auto-refilled from pharmacy could bring a better control of work schedule, as well as an increase in the amount of returned drug when the drug therapy has changes.

\section{INPATIENT MEDICATION ORDER ENTRY (IPMOE)}

The Hospital Authority has been using an electronic medication system, Inpatient Medication Order Entry (IPMOE), to replace the hand-written order form since 2010. IPMOE is a modernized module which is gradually implemented in 15 acute hospitals across all the 7 clusters. [3] It is an internally built It system with a closed loop for in-patient drug management. This integration system allows frontline healthcare professionals to login the IPMOE via different channels, like desktop computers, computers on wheel, or mobile devices such as iPads and tablets. The mobile apparatus improves the efficiency of administration process and minimizes prescription and administration errors, thus improving both patient safety and users' experience.

The module provides real-time accessibility to patients' medication profiles, without paper prescriptions. Medical practitioners can prescribe electronically via IPMOE without using manual records and thus avoiding errors resulting from poor handwriting. Pharmacists can check and vet prescriptions in IPMOE and dispense through the desktop computers equipped with HA secured intranet, supported with barcode scanners, printers and label printers. Nurses are able to receive and read the patient's record at the point of care by using mobile terminals in inpatient areas (Fig. 3).[10] The system helps in streamlining workflow, improvement of efficiency, integration of Medication Decision Support knowledge, standardization of medication ordering line, reduction of medication errors, enhancing communication between caregivers and improving medication documentat

\section{FIGURE 3: FLOWCHART OF INPATIENT MEDICATION ADMINISTRATION PROCESS (IPMOE)}

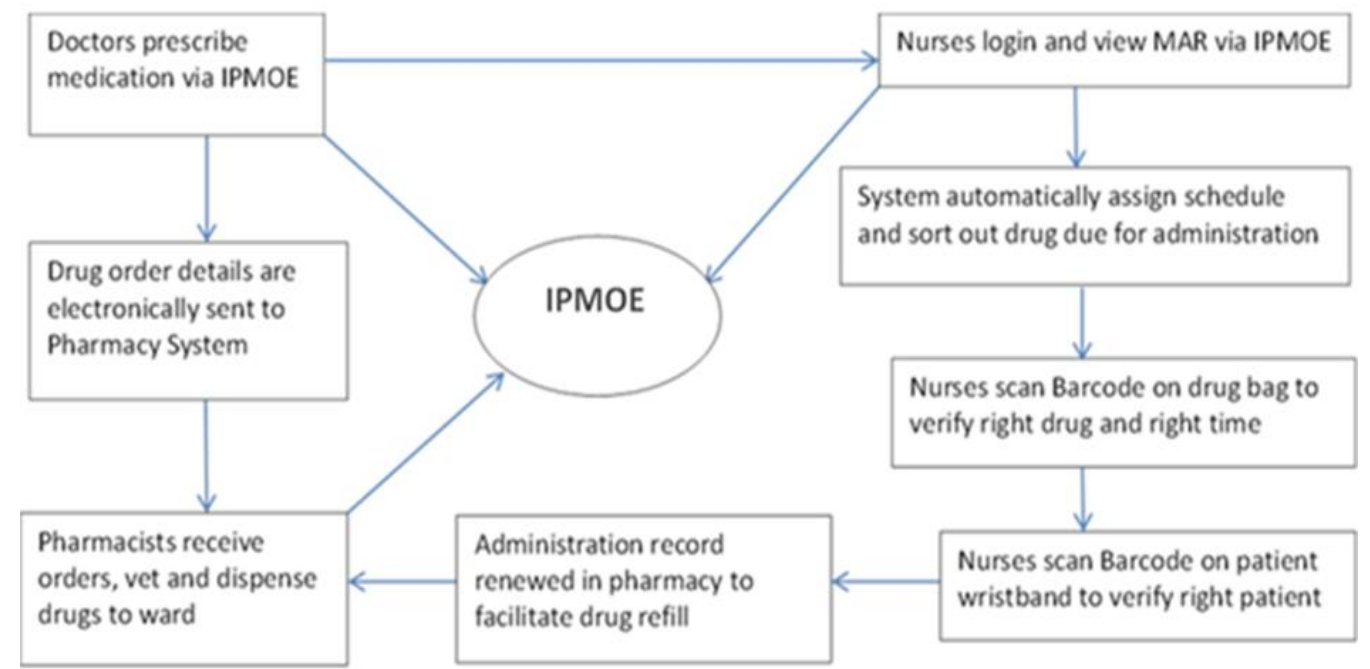

Source: [10] Leung CB. Implementation Journey of Inpatient Medication Order Entry Project in Prince of Wales Hospital [Internet]. 2015 [cited 2019 Nov 5 ]. Available from https://www3.ha.org.hk/haconvention/hac2015/proceedings/downloads/S12.4.pdf 


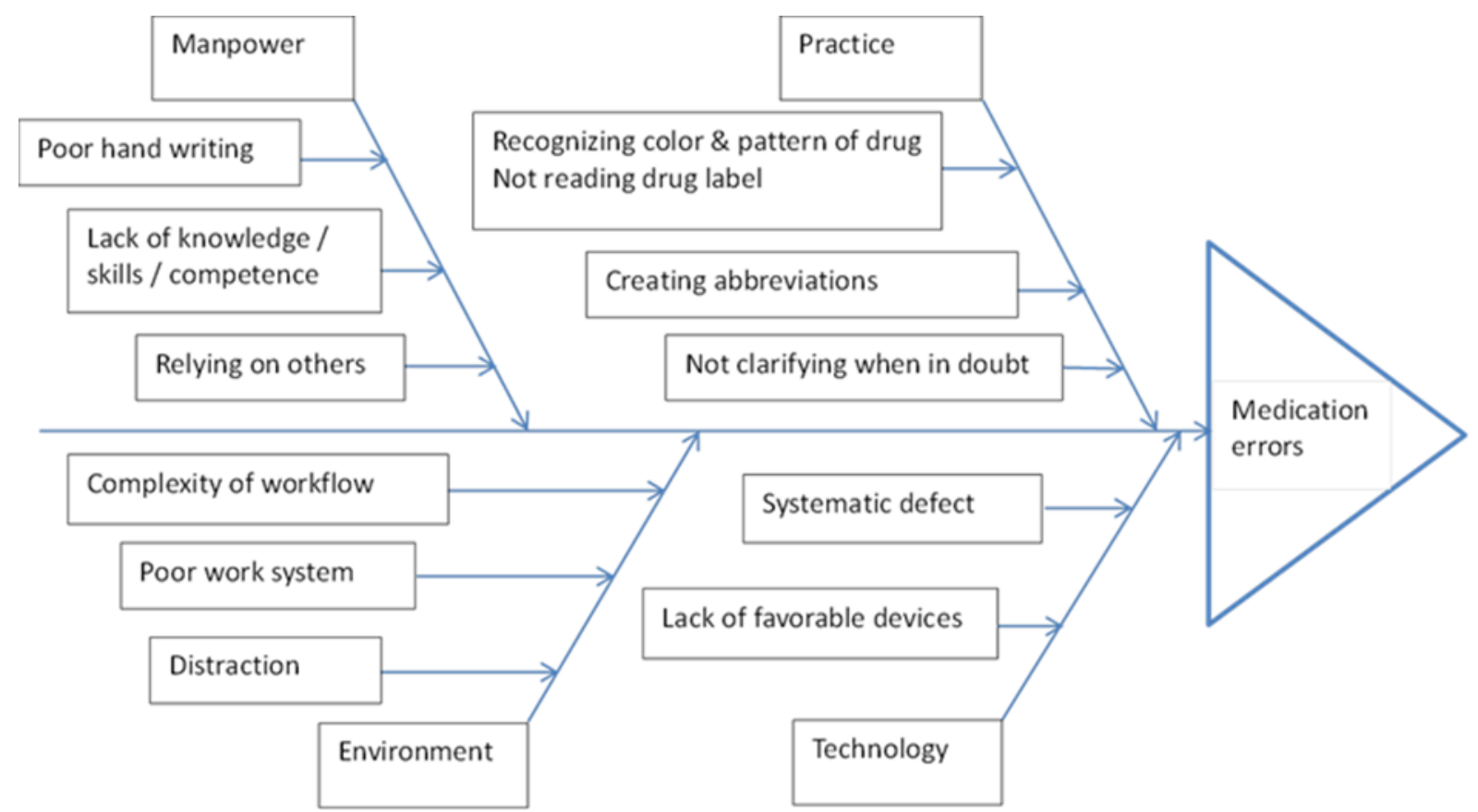

Source: [15] Hou N, Tang H. Analysis of Medication Errors in Provincial Hospital. Biomedical Journal of Scientific \& Technical Research. 2017; 1 (4).

\section{FACTORS ASSOCIATED WITH MEDICATION ERRORS}

\section{PROVIDERS}

The poor handwriting orders by doctors would definitely affect the transcription by pharmacists and the administration procedures in the ward by nurses, leading to increased risk and frequency of medication incidents. Ambiguous handwriting orders make it difficult for pharmacists to enter the record into the pharmacy system. Such and incomplete orders often consume extra time in returning to the physicians for clarification and would increase the waiting time.

\section{WORKING ENVIRONMENT}

Heavy workload in public hospitals is one of the sources of stress for staff, particularly the medical practitioners, pharmacists and nurses. Doctors often work under urgent and critical circumstances, resulting in making errors more frequently.[11] Fatigue from high demand of work may affect the performance of healthcare professionals, leading to the poor recognition and assessment of orders from doctors and pharmacists. In addition, poorly designed working area is also a risk factor. For example, the drugs stored in ward stock should be managed regularly depending on the usage in each ward. However, nurses are required to do a large amount of administrative paperwork, and so time spent in checking the quantity and quality of medications is reduced. Samaranayake has mentioned that distractions from environment and workflow deficiencies are factors which could cause staff to make mistakes in computer entry, increasing the chance of medication errors.[7]

\section{PRESCRIBING PRACTICE}

Abbreviations are commonly used in prescribing practice. The adoption of special codes and terms in prescriptions is a universal method used by doctors in handwritten prescription, by pharmacists and dispensers in transcription and in picking drugs in pharmacy, and by nurses in medication administration. However, there are some unapproved abbreviations in the "Do Not Use" list, because similar abbreviations would cause confusion to those working in other units. The list helps to minimize medication incidents arising from the misuse of unapproved or unconventional abbreviations.[12] For instance, "I.A." is indicating the route of drug administration. This abbreviation may have a misinterpretation between "interarterial" and "intra-articular", leading to errors and potential harm.

Verbal orders are sometimes used by doctors during emergency situations, but this is not recommended when orders are not put into the system. Phone instructions could affect the content and context of the verbal orders, arising 
from spelling errors in drugs with similar names, using nonstandardized abbreviations and terminology, differences in language skills and clarity of communications of the speaker, and even the noise level in the environment as well.[13]

Inappropriate working procedures are the most common cause in mixing up the barcoded patient identification labels.[7] The barcode labels are used to eliminate 'wrongpatient' errors. However, the errors still exist when staff do not follow the proper procedures. It has been found that some nurses may 'violate' the rules or use 'workarounds' to overcome the obstacles associated with using the barcode technology in drug administration. [7] A study in 2017 illustrated that workarounds were used in $66 \%$ of medication administrations in hospitals using barcodeassisted technology.[14] When staff encounters unexpected situations or impractical steps, they may find ways to circumvent the correct procedures, increasing the opportunity to making errors. Therefore, simple systems allow flexibility in workplaces for nurses and frontline staff, while setting standards and rules in working procedures helps to ensure the accuracy of dispensing medicine.

\section{COMPUTERIZED INFORMATION SYSTEM}

Poor interface between users and the computer system is a common cause for technology-related errors.[7] Knowledge of staff in using a new system and computer over-reliance are also contributing to errors. Out-dated modules with unnecessary data set may create inferior user interfaces. For example, when using infusion pumps, the deficiency of key pad usually generate errors. As a result, nurses would enter doses and flow rates which are not intended.[7] It may lead to extra doses given to patients when a missed decimal dot or an additional zero is entered, and this would increase the risk to patient safety. Introducing a safe system, counter checking by the device operator and verification by another nurse are measures to avoid such errors.

Quality Management in hospitals is important for assessing and improving the quality of healthcare services. A simplified model named '4P Excellence Model' provides a recommended structure to build quality into 4P: people, partnership, process of work and products / service products.[16] This strategy should be multi-directional and be implemented in both top-down and bottom-up approaches. Danish Hospitals have adopted the '4P
Excellence Mode' with 'European Excellence Model' for evaluating and diagnosing the level of excellence in healthcare services. A number of key performance indicators (KPIs) are selected in areas of leadership, people management, partnership and resources, processes and products/ services results.[16]

In Singapore, The National University Hospital has fully adopted the operation of an end-to-end 'closed-loop' electronic system called the Closed Loop Medication Management System (CLMMS) in the inpatient setting. CLMMS is composed of Electronic Inpatient Medication Record, Clinical Decision Support System, Inpatient Pharmacy Automated System and Electronic Medication Administration Record System.[17] The improvement outcome is proved by KPIs, including the following: (1) $80 \%$ of medication orders are stocked in cabinet, (2) $91 \%$ of medications administered are barcoded, (3) safe picking of medications with reduced errors per 100 days, (4) correct medications administration with prevented errors per 100 patient days, (5) secure medication storage, (6) improved work efficiency, (7) redeployment to patient centric activities, and (8) streamlined the restocking process with real time inventory. It has indicated that the Closed Loop Medication Management System is a successful tool to perform the medication distribution along the inpatient system in terms of achieving the four 'rights' of medication safety, which are the right patient, the right drug, the right dose and the right time.[17]

\section{RECOMMENDATIONS}

\section{AUTOMATION}

Implementation of Automated Pharmacy Distribution Systems is the key in moving towards closed loop medication management systems.[18] Some procedures in the preparation and distribution of medications can be automated. For example, in-pharmacy robotics can perform the storing and picking of medicines and the labelling stage of the dispensing process. The Automated Tablet Dispensing and Packaging System pack tablets and capsules into barcoded unit doses which facilitate the medication administration in wards.[17] Each unit dose of medicine, for tablet and capsule dosage form, is separately packed with a protective sealed unit. To facilitate the closed loop medication management, these units are barcoded and labelled with the drug name, strength, batch number and expiry date.[18] To monitor the drug inventory, a controlled substances cabinet located in 
the pharmacy is a computerized drug storage device to track individuals who access to the cabinet and drug distribution.[18]

\section{CLINICAL PHARMACISTS}

Interventions by pharmacists are useful in intercepting errors, and so clinical pharmacy services should be introduced as a strategy in public hospitals.[7] Clinical pharmacists can assist nurses in the pharmaco-therapeutic information during medication administration in wards. Double checking by another colleague helps to prevent prescribing errors during drug administration, without just depending on the surveillance of nurses. Clinical pharmacists may present valuable data on common medication errors, which help in implementing technological innovations to monitor or strengthen the medication safety.[7] They may also educate patients about their drugs, whereby patients could be more knowledgeable regarding their own medication therapies.

\section{TECHNOLOGICAL INNOVATIONS}

Modifying technologies in barcode assisted medication administration technology and smart infusion pumps can help intercepting drug administration errors. A closed-loop electronic medication administration system with barcode verifying technology significantly reduces timing and nontiming errors.[19]

Automated Dispensing Cabinets (ADCs) are suggested to store ward stock medications in the wards.[20] It is a computer-controlled system that can interface with external devices. The clinical medication orders could be reviewed by the pharmacist before drug administration by the nurses. ADCs are able to interface with barcode technology to function in the restocking process and tracking dispensed medications automatically. In addition, when it is linked with the point-of-care barcode system, it can help to ensure matching the prescribed drugs and selected drugs electronically, so as to enhance the efficiency of drug dispensing at the bedside and reduce the 'wrong drug' errors.

\section{POP-UP ALERT IN IPMOE SYSTEM}

The enhancement in IPMOE with a pop-up message will alert staff during prescribing, dispensing and administration. In the prescribing module, patient's allergy information should be indicated clearly in a pop-up message in a red box when prescribers are entering the patient's medication records.[3] For repeat prescription of "fixed period" and "single use" medications, nurses should check for the popup alert which is displayed on the electronic patient record, and ask doctors or clinical pharmacists for confirmation. For prolonged prescriptions of a certain drug, an alert pop-up screen should be shown to remind the physician to provide the expected prescription duration.

In the drug administration module, it needs a pop-up window to call the attention from nurses in administering the criteria-based medication and the record should be listed in the drug administration record to facilitate further treatment or clinical management.[3] When a drug is not suitable for patient, it should be marked as "Omit" by nurses to present a clear documentation and record. Moreover, improving the IPMOE display according to ward columns is useful for the frontline staff in drug administration, such as changing the remarks to 'Withhold' or 'SelfAdmin' instead of 'Prescription changed' and 'Unscheduled' column.

\section{CONCLUSION}

It is imperative to achieve medication safety in hospitals during every process, including prescribing, transcribing, dispensing and administering in order to reduce the medication incidents. Effective strategies to ensure medication safety include implementing clinical pharmacists in wards to work with nurses, utilizing automated system and technical devices in pharmacy enhance the efficiency and accuracy of medication preparing and dispensing. Upgrading the IPMOE alert system is critically important for the working process in drug prescription and administration.

\section{ACKNOWLEDGEMENT}

The work described in this paper was partially supported by a grant from the Research Grants Council of the Hong Kong Special Administrative Region, China (Project Reference No.: UGC/IDS24/18).

\section{References}

1. Samaranayake NR, Cheung STD, Chui WCM, Cheung $B M Y$. The pattern of the discovery of medication errors in a tertiary hospital in Hong Kong. International Journal of Clinical Pharmacy. 2013; 35: 432-438.

2. World Health Organization. Medication Errors: Technical Series on Safer Primary Care [Internet]. 2016 
[cited 2019 Nov 5]. Available from

https://apps.who.int/iris/bitstream/handle/10665/2522

74/9789241511643-

eng.pdf;jsessionid=BDBFDEDF2F2EE91 EAE222F640342D

153 ? sequence $=1$

3. Hospital Authority, Hong Kong Special Administrative Region. Quality and Safety Annual Report 2018 [Internet]. 2019 [cited 2019 Nov 5]. Available from http://www.ha.org.hk/haho/ho/psrm/EQnSReport2018 .pdf

4. National Coordinating Council for Medication Error Reporting and Prevention. What is a medication error? [homepage on the Internet]. c2019 [cited 2019 Nov 5]. Available from https://www.nccmerp.org/aboutmedication-errors

5. Reason J. Human error: models and management. British Medical Journal. 2000; 320 (7237): 768-770.

6. Yousef $N$, Yousef F. Using total quality management approach to improve patient safety by preventing medication error incidences. BMC Health Service Research. 2017; 17, 621.

7. Samaranayake NR. Medication safety in hospitals: medication errors and interventions to improve the medication use process. [unpublished dissertation]. University of Hong Kong, Pokfulam, Hong Kong Special Administrative Region. 2013. [cited 2019 Nov 5] Available from http://dx.doi.org/10.5353/th_b5108672

8. Chiang SC. In-Patient Medication Order Entry System contribution of pharmacy informatics [Internet]. 2015 [cited 2019 Nov 5]. Available from http://www3.ha.org.hk/haconvention/hac2015/proce edings/downloads/S12.1.pdf

9. Hospital Authority, Hong Kong Special Administrative Region. Hospital Authority Annual Report 2013-2014 [Internet]. 2014 [cited 2019 Nov 5]. Available from https://www.ha.org.hk/ho/corpcomm/ar201314/pdf/F ullSet.pdf

10. Leung CB. Implementation Journey of Inpatient Medication Order Entry Project in Prince of Wales Hospital [Internet]. 2015 [cited 2019 Nov 5]. Available from

https://www3.ha.org.hk/haconvention/hac2015/proc eedings/downloads/S12.4.pdf

11. Patel R, Bachu R, Adikey A, Malik M, Shah M. Factors Related to Physician Burnout and Its Consequences: A Review. Behavioral Sciences. 2018; 8(11): 98.
12. Hospital Authority, Hong Kong Special Administrative Region. Risk Alert, Issue 28 [Internet]. 2013 [cited 2019 Nov 5]. Available from https://www.ha.org.hk/haho/ho/psrm/HARA28th.pdf

13. Moghaddasi H, Farahbaksh M, Zehtab H. Verbal Orders in Medicine: Challenges; Problems and Solutions. JOJ Nursing \& Health Care. 2017; 1 (5).

14. Van der Veen W, van den Bemt PM, Wouters H, Bates DW, Twisk JW, de Gier JJ, Taxis K. Association between workarounds and medication administration errors in bar-code-assisted medication administration in hospitals. Journal of the American Medical Informatics Association. 2017; 25(4): 385-392.

15. Hou N, Tang H. Analysis of Medication Errors in Provincial Hospital. Biomedical Journal of Scientific \& Technical Research. 2017; 1(4).

16. Dahlgaard JJ, Pettersen Jp, Dahlgaard-Park SM. Quality and lean health care: A system for assessing and improving the health of healthcare organisations. Total Quality Management \& Business Excellence. 2011; 22(6): 673-689.

17. Foo GTT, Tan $\mathrm{CH}$, Hing WC, Wu TS. Identifying and quantifying weaknesses in the Closed Loop Medication Management System in reducing medication errors using a direct observational approach at an academic medical centre. Journal of Pharmacy Practice and Research. 2017; 47(3): 212220.

18. The Society of Hospital Pharmacists of Australia. Factors to consider for the implementation of Automated Pharmacy Distribution Systems in Hospitals and Health Services [Internet]. 2019 June [cited 2019 Nov 5] Available from https://www.shpa.org.au/sites/default/files/uploadedcontent/website-content/Fact-sheets-positionstatements/automation_practice_update.pdf

19. Poon EG, Keohane CA, Yoon CS, Ditmore M, Bane A, et al. Effect of bar-code technology on the safety of medication administration. The New England Journal of Medicine. 2010; 362: 1698-1707.

20. Grissinger M. Safeguards for Using and designing automated dispensing cabinets. A Peer-Reviewed Journal for Managed Care and Hospital Formulary Management. 2012; 37(9): 490-491. 